the importance of remaining vigilant about nephritis risk in patients of diverse demographics five years and more after diagnosis with SLE.

Funding Source(s): Mallinckrodt Research Fellowship, NIH: T32DK007257,UL1TR001105,U54GM104938,P30AR053483, U01AI101934,U19AI082714

\section{BARICITINIB INHIBITS B CELL ACTIVATION AND AMELIORATES MURINE LUPUS}

Jaeseon Lee*, SeGwang Jang, Seung-Min Hong, SeungYe Baek, Sung-Hwan Park, SeungKi Kwok. The Catholic University

\subsection{6/lupus-2019-Ism.22}

Background B cells carry out central roles in the pathogenesis of SLE by triggering tissue inflammation through autoreactive antibody-dependent responses. Baricitinib is a JAK1/2 inhibitor that blocks the downstream of multiple cytokine receptor signaling implicated in lupus pathogenesis.

Methods We investigated the effect of Baricitinib to the differentiation of $\mathrm{B}$ cells in vitro. And female MRL/lpr mice were administered baricitinib $(10 \mathrm{mg} / \mathrm{kg})$ or vehicle $(0.5 \%$ methyl cellulose) by gavage twice daily for 8 weeks, when determined serum levels of anti-ds DNA antibodies and pro-inflammatory cytokines (BAFF, IL-6 and IL-17), proteinuria, renal inflammation and IgG-C3 deposit. In vitro, Baricitinib suppressed the mRNA expression of AID, Bcl6, XBP-1, IRF4 and the production of $\operatorname{IgG}$ in B cells.

Results In MRL/lpr model, Baricitinib-treated mice showed reduced levels of anti-dsDNA antibodies, proteinuria, and cytokines as compared with those of control mice. In addition, Baricitinib prevented renal pathology, as judged by changes in the histopathological scores of glomeruli with PAS stain and IgG-C3 deposition by confocal microscope. Finally, Baricitinib treatment significantly inhibited downstream of Jak/ STAT signals in CD19 + B cells from spleen. In conclusion, this study revealed the regulatory effects of baricitinib on B cells and ameliorates murine lupus.

Conclusions These results indicate that Jak inhibitors have the potential therapeutic approach for SLE.

Funding Source(s): None

\section{TREATING LUPUS-PRONE MICE WITH A COENZYME Q10 ANALOG DECREASES DISEASE PARAMETERS INDICATING A THERAPEUTIC ROLE IN SYSTEMIC LUPUS ERYTHEMATOSUS}

${ }^{1}$ Luz Blanco, ${ }^{2}$ Hege Lynum Pedersen*, ${ }^{1}$ Yaima L Lightfoot, ${ }^{1}$ Nickie Seto, ${ }^{1}$ Carmelo CarmonaRivera, ${ }^{1}$ Yudong Liu, ${ }^{3}$ Victoria Hoffman, ${ }^{4}$ Peter ST Yuen, ${ }^{1}$ Mariana J Kaplan. ${ }^{1}$ NIH NIAMS ${ }^{2}$ NIH NIAMS/UiT-The Arctic University of Norway; ${ }^{3}$ Office of the Director, Division of Veterinary Resources, Diagnostic and Research Services Branch, NIH; ${ }^{4}$ Renal Diagnostics and Therapeutic Unit, Kidney Diseases Branch, National Institute of Diabetes and Digestive and Kidney Diseases, NIH

\subsection{6/lupus-2019-Ism.23}

Background Immune dysregulation and organ damage are characteristic of systemic lupus erythematosus (SLE). We previously reported that mitochondrial reactive oxygen species (ROS) and the release of oxidized mitochondrial DNA (mtDNA) may be involved in autoimmunity and contribute to the generation of type I IFN responses, important mediators in the pathogenesis of SLE. Mitochondrial dysfunction

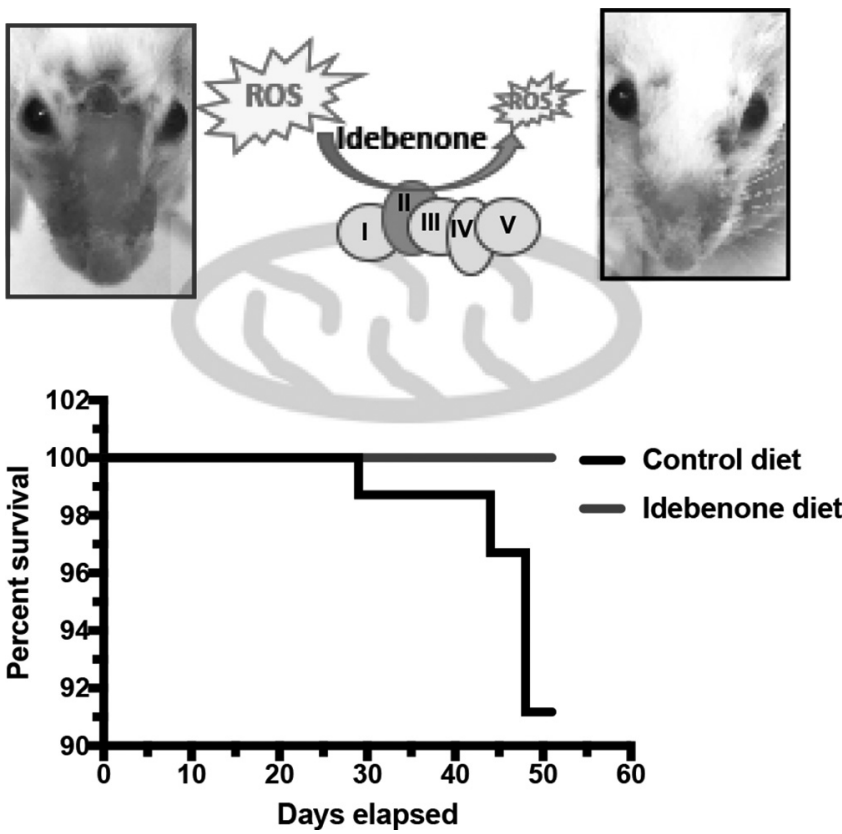

Abstract 23 Figure 1 Effects of idebenone treatment on lupus-prone mice. Idebenone treatment showed improvements such as reduction of skin ulcers and attenuation in mortality

and an imbalance in ROS production may lead to oxidative stress, mitochondrial lipid, protein and DNA modifications, and alterations in the production of adenosine triphosphate (ATP). Idebenone is a coenzyme Q10 synthetic analog with antioxidant properties previously tested in humans to treat different diseases where mitochondrial function is compromised. This study aimed to assess if idebenone mitigates murine lupus.

Methods Two different doses of idebenone were given orally to $\mathrm{MRL} / \mathrm{lpr}$ mice for 8 weeks. At euthanasia, treated and untreated mice were analyzed and compared for clinical, immunologic and metabolic parameters including autoantibody generation, proteinuria, kidney function and pathology, renal immune complex deposition, endothelium-dependent vasorelaxation, assessment of pro-inflammatory gene expression, quantification and characterization of neutrophil extracellular trap (NET) formation and mitochondrial ROS, measurement of complex II-specific activity and cardiac lipid peroxidation.

Results Idebenone was well tolerated and led to significant attenuation in mortality and an improvement of glomerular inflammation, renal function, skin ulcers and splenomegaly. The levels of proinflammatory cytokines and inflammasomerelated genes were significantly decreased by idebenone. In addition, we observed inhibition of NET formation both in lupus mice bone marrow neutrophils and in neutrophils from lupus patients. Enhanced complex II-specific activity and mitochondria specific gene transcription were shown in cells isolated from idebenone-treated lupus-prone mice, suggesting improved mitochondrial function and reduction of oxidative stress. Idebenone-treated mice also displayed reduced heart lipid peroxidation.

Conclusions Idebenone improves murine lupus. These results suggest that idebenone and other drugs that improve mitochondrial function may have a therapeutic role in SLE.

Funding Source(s): This study was supported by the Intramural Research Program, NIAMS/NIH (ZIA AR041199) and the Alliance for Lupus Research. 